\title{
Asiye Aslan
}

Bandırma Onyedi Eylül University, aaslan@bandirma.edu.tr, Balıkesir-Turkey

\begin{tabular}{l|l|l}
\hline \hline DOI & http://dx.doi.org/10.12739/NWSA.2021.16.2.1A0470 \\
\hline ORCID ID & $0000-0002-1173-5008$ \\
\hline Correspoding Author & Asiye Aslan \\
\hline \hline
\end{tabular}

\section{INVESTIGATION OF THE EFFECT OF OPTIMUM INSULATION THICKNESS OF BUILDING EXTERIOR WALLS ON ENERGY CONSUMPTION AND REDUCTION OF EMISSIONS}

\section{ABSTRACT}

The heating and cooling load of buildings gets reduced by preventing heat transfer to the external environment using exterior wall insulation. In addition to the saving achieved by reducing fuel consumption, the amount of harmful gases emitted into the atmosphere also gets reduced and a positive contribution is made to the environment. It is a known fact that 85\% of $\mathrm{CO}_{2}$ emission in Turkey is caused by the energy sector in Turkey. In this study, the optimum insulation thickness of exterior walls and emission per unit area was calculated for 81 provinces in Turkey. The Life Cycle cost method was used as the approach. As a result, it was determined that while the optimum insulation thickness varied between $0.024 \mathrm{~m}$ (İçel) and $0.149 \mathrm{~m}$ (Ardahan), a 75\%-80\% reduction can be achieved in emissions. Additionally, energy savings and payback periods based on optimum insulation thickness in heating and cooling for provinces selected from five different climatic zones (Antalya, Istanbul, Ankara, Kayseri, and Erzurum) were calculated and compared. In the selected provinces, the highest energy savings and the shortest payback period were obtained for Erzurum as $46.93 \$ / \mathrm{m}^{2}$ and 1.33 years.

Keywords: Degree Days, Energy Saving, Optimum Insulation Thickness, Greenhouse Gas Emission, External Walls

\section{INTRODUCTION}

Economic crises and depletion of energy resources across the world have made efficient use of energy necessary. More than three quarters of the energy used in buildings is used for heating and cooling. Lack of or insufficient insulation causes loss of some energy in buildings. However, it is possible to prevent this energy waste to a great extent by insulating the outer walls, which are the most heatlosing surface in buildings. Other important benefits of insulation are thermal comfort and environmental protection. Thermal insulation establishes an ideal balance between the human body and environment temperatures in buildings with insulation. A reduced fuel consumption thanks to building insulation also reduces the amount of greenhouse gas emissions, contributing to the protection of environment. In recent years, several studies have been conducted especially on energy conservation through insulation in buildings. Kürekçi determined the optimum insulation thickness by making separate calculations for 81 provinces in Turkey according to heating and cooling degree days and using four different fuels and three different insulation materials. Accordingly, net energy conservation increased, payback periods decreased for natural gas, coal, fuel-oil and LPG, respectively [1]. Açıkkalp and Yerel Kandemir presented an alternative and new method,

How to Cite:

Aslan, A., (2021). Investigation of The Effect of Optimum Insulation Thickness of Building Exterior Walls on Energy Consumption and Reduction of Emissions. Engineering Sciences, $16(2): 64-77$, DOI: 10.12739/NWSA.2021.16.2.1A0470. 
called the Combined Economic and Environmental Method, in which economic and environmental impacts were combined to determine the optimum insulation thickness. By using this method, they analyzed the use of rock and glass wools on outer walls in Bilecik, Turkey, and determined the optimum points for an economic and environmental approach [2].

Alsayed and Tayeh calculated the optimum insulation thicknesses on different degree days in Palestine by using LPG for winter and electricity for summer, and concluded that insulation type and degree day value had the most important effect on optimum insulation thickness [3]. Şencan Şahin and Dikmen determined the optimum insulation thickness for different fuel types to save energy during heating buildings in winter and cooling them in summer in Denizli, Turkey, taking into account $\mathrm{CO}_{2}$ emissions. They found that the optimum insulation thickness varied between 0.012-0.031m for heating in winter and between 0.009-0.022m for cooling in summer [4]. Karakaya performed an analysis of optimum insulation thickness, energy conservation, payback period and environmental impact during heating and cooling of buildings in Batman, Turkey. The author used four different wall components, three different types of fuel, and two different insulation materials, and determined that $\mathrm{CO}_{2}$ and $\mathrm{SO}_{2}$ emissions decreased by $77.00 \%$ in optimum insulation thickness compared to the uninsulated situation [5].

Ozel studied thermal, economic, and environmental effects for different wall directions in insulated buildings under dynamic thermal conditions in Kars, one of the cities with the lowest average air temperatures in Turkey. In the study, calculations were made for two different wall structures and two different insulation materials. For the heating season, the lowest heating load, optimum insulation thickness, and energy savings were achieved in the south-facing wall, while the highest heating load, optimum insulation thickness, and energy savings were achieved for the north-facing wall. When using 9 $\mathrm{cm}$ insulation, an 85\% reduction in emissions was detected for all wall orientations [6]. In their study, Küçüktopçu and Cemek calculated the optimum insulation thickness, energy savings, payback period, and $\mathrm{CO}_{2}$ emission rates for poultry shelters in Antalya, Samsun, Ankara, and Erzurum provinces of Turkey. Five different energy sources (coal, fuel oil, natural gas, LPG, and electricity), two different insulation materials, Extruded Polystyrene (XPS), and Expanded Polystyrene (EPS) were used in the study. The lowest $\mathrm{CO}_{2}$ emission reduction value (39.82\%) was obtained when XPS was used as the optimum insulation material and natural gas was used as the energy source in Antalya, while the highest value was obtained when EPS and LPG (83.98\%) were used in Erzurum [7].

In their study, Nyers et al. analyzed the optimum energy and economical thickness of the thermal insulation layer for the outer walls. The analysis was carried out by implementing a new "investment savings" method. The mathematical model consisted of energy and economics. Analytical-numerical and graphical-numerical methods have been implemented to solve the mathematical model whose optimization criterion was the minimum payback period of the initial investment. With the developed mathematical model, the optimum thermal insulation layer thickness was obtained in Serbia in 2014 for energy and economic conditions. As an important result of the study, a 4.86 times increase in thickness is achieved with only a 1.69 times increase in investment [8]. Muddu et al. calculated the optimum insulation thickness, annual energy consumption, payback period, and $\mathrm{CO}_{2}$ emissions for typical walls in 25 regions of Ireland. In the study, they reported that the wall type, materials, configuration, insulation type, and the type of heat 
energy all had significant effects on the annual cost. The calculated optimum insulation thickness in Ireland varied by $30 \%$ as a result of the increase in the number of heating days from low to high [9]. Yuan et al. determined the optimal thermal resistance of insulation materials for residences in six climate zones of Japan, with a combination of four different insulation materials and four different fuel sources. They calculated the energy cost and payback period of the outer walls per unit area. The degree-day (DD) method was used in calculations. In the study, the optimum thermal resistance decreased from Climate Zone I to VI (from low latitude to high latitude). However, the payback period for insulated buildings had an opposite trend [10]. Liu et al. presented a combination of heat and moisture transfer model that takes into account the effect of moisture transfer on heat transfer to calculate the cooling and heating transmission load. They determined the optimum insulation thickness of the exterior walls using the P1-P2 economic model. Changsha, Chengdu, and Shaoguan were chosen as the sample cities in China. The results showed that the optimum thickness of Extruded Polystyrene (XPS) ranged from 0.053 to $0.069 \mathrm{~m}$ and the optimum thickness of Expanded Polystyrene (EPS) ranged from 0.081 to $0.105 \mathrm{~m}$ [11]. Sabapathy et al. performed an energy-saving analysis by using a numerical analysis for three building shell configurations in five different climate zones of India. The insulating potential of straw, which is an agricultural waste in the context of India's broad climate, was the focus of this study. They concluded that energy savings in the range of $67-96 \%$ can be achieved in the different climatic zones by introducing straw to the outer shell only $10 \mathrm{~cm}$ in thickness [12].

\section{RESEARCH SIGNIFICANCE}

Carbon dioxide has the largest share of greenhouse gases. The most important source of carbon dioxide is fossil fuels. It is essential and crucial to use optimum insulation thickness in buildings for reducing fuel consumption and emission values. In this study, optimum insulation thicknesses were determined for building exterior walls located in 81 provinces in Turkey, and fuel consumption and emission values per unit area were calculated depending on the insulation thickness. When the literature was examined, no such study including all the provinces in Turkey was encountered. In this respect, the study is expected to contribute to the literature. Two different insulation materials and two different commonly used fuels were chosen in the study. The degree day method was used to analysis. However, this study examined the optimum insulation thickness of external walls and energy conservations and payback periods, comparing provinces from five different climate zones in Turkey and taking into account the heating of buildings in winter and cooling in summer. Electricity was used as an energy source for cooling. Energy conservations and payback periods were calculated for a 10-year lifetime.

\section{MATERIAL AND METHOD}

\subsection{Optimum Insulation Thickness on External Walls}

Optimum insulation thickness for external walls in buildings varies according to number of degree days, outside temperature, type of fuel, type of thermal insulation material, and economic criteria such as lifestyle, inflation and interest rate. In this study, lifecycle costing (LCC) method was used for the analysis of external walls [13, 14 and 15]. Heating and cooling degree days values are obtained from Ref. [1]. In the analysis, two different fuels (natural gas and coal) for heating and electricity for cooling and two different 
insulation materials (XPS and EPS), which are widely preferred in external wall insulation in Turkey, were used according to the sheathing method. Table 1 shows the properties and costs of natural gas and coal fuels used in heating and the cost of electricity used in cooling. Table 2 presents the properties of XPS and EPS insulation materials.

Table 1. Properties of fuels and system efficiency [16 and 17]

\begin{tabular}{|l|c|cc|c|}
\hline & Cost & Lower HeatingValue, $\mathrm{H}_{\mathrm{u}}$ & System Efficiency $(\%)$ \\
\hline Natural Gas & $0.2868 \$ / \mathrm{m}^{3}$ & $34.542 \times 106$ & $\mathrm{~J} / \mathrm{m}^{3}$ & 93 \\
\hline Coal & $0.1921 \$ / \mathrm{kg}$ & $25.122 \times 106$ & $\mathrm{~J} / \mathrm{kg}$ & 65 \\
\hline Electricity & $0.1252 \$ / \mathrm{kWh}$ & \multicolumn{3}{|c|}{$2.5 \mathrm{COP})$} \\
\hline
\end{tabular}

Table 2. Properties of insulating materials [18]

\begin{tabular}{|c|c|c|c|}
\hline & Heat Conductio & Coefficient, $\lambda(\mathrm{W} / \mathrm{mK})$ & Cost (\$) \\
\hline XPS (Extruded Polystyrene) & & 0.032 & 85 \\
\hline EPS (Expanded Polystyrene) & & 0.035 & 50 \\
\hline
\end{tabular}

\subsection{Calculation of the Heat Load}

The heat losses in buildings generally occur through external walls, windows, ceiling, floor and air infiltration. The heat transfer coefficient $U\left(\mathrm{~W} / \mathrm{m}^{2} \mathrm{~K}\right)$ of a wall that includes a layer of insulation is given by:

$$
U=\frac{1}{R_{i}+R_{W}+R_{\text {ins }}+R_{O}}
$$

Where $R_{i}$ and $R_{0}$ are the inner and outer air-film thermal resistances, respectively. $R_{w}$ is the total thermal resistance of the wall layers without insulation. The thermal resistance of the insulation layer $R_{\text {ins }}$ is given by:

$$
R_{\text {ins }}=\frac{x}{\lambda}
$$

Where $x$ and $\lambda$ are the thickness and the thermal conductivity of the insulation material, respectively. If $R_{w t}$ is the total the wall thermal resistance excluding the insulation layer resistance, equation (1) can be rewritten as:

$$
U=\frac{1}{R_{w t}+R_{\text {ins }}}
$$

The heat loss from unit surface of external wall:

$q=U \Delta T$

Where $U$ is the overall heat transfer coefficient. The annual heat losses from unit area can be approximately calculated depending on the degree-days number as the following:

$$
q_{A}=86400 D D U
$$

Where $D D$ is the degree days. The annual energy requirement for heating $\left(E_{A}\right)$ can be obtained approximately by dividing the annual heat loss to the efficiency of the heating system $\left(\eta_{s}\right)$ :

$$
\begin{aligned}
& E_{A}=\frac{86400 D D}{\left(R_{w t}+\frac{x}{\lambda}\right) \eta_{s}} \\
& m_{f A}=\frac{86400 D D}{\left(R_{w t}+\frac{x}{\lambda}\right) H_{u} \eta_{s}}
\end{aligned}
$$

The annual heating cost $C_{A}, H$ (\$ $/ \mathrm{m}^{2}$-year) per unit area can be calculated by the equation (8):

$$
C_{A, H}=\frac{86400 H D D C_{f}}{\left(R_{w t}+\frac{x}{\lambda}\right) H_{u} \eta_{s}}
$$

Where $C_{f}$ is fuel cost $(\$ / \mathrm{kg})$ and $H_{u}$ is the lower heating value of the fuel $\left(\mathrm{J} / \mathrm{kg} ; \mathrm{J} / \mathrm{m}^{3}\right)$. The annual cooling cost per unit area can be calculated using equation (9). COP is the performance coefficient of the cooling system and it was taken as 2.5 in this study [1]:

$$
C_{A, C}=\frac{86400 C D D C_{f}}{\left(R_{w t}+\frac{x}{\lambda}\right) C O P}
$$




\subsection{Optimum Insulation Thickness Calculation}

The LCC is one of the methods to calculate the optimum insulation thickness. Total heating cost is evaluated together with the present-worth factor $P W F$ for the lifetime of $N$ years. The PWF depends on the inflation rate (g), and the interest rate (i). According to the interest and inflation rates, PWF is defined as below:

i>g then,

$i<g$ then,

$$
r=\frac{i-g}{1+g}
$$

$P W F=\frac{(1+r)^{N}-1}{r(1+r)^{N}}$

$$
r=\frac{g-i}{1+i}
$$

$N$ life was taken as 10 years and annual interest (i) and inflation $(g)$ rates were taken as $8.25 \%$ and $12.66 \%$, respectively according to 2020 data [19 and 20].

The total heating cost of the insulated building is given by:

$C_{t}=C_{A} P W F+C_{I} x$

$C_{t}=\frac{86400 H D D C_{f} P W F}{\left(R_{w t}+\frac{x}{\lambda}\right) H_{u} \eta_{s}}+C_{I} x$

Where $C_{I}$ is the cost of insulation material in $\$ / \mathrm{m}^{3}$ and $x$ is the insulation thickness in $\mathrm{m}$. The optimum insulation thickness is obtained by minimizing equation (12). Hence, the derivative of $C_{t}$ with respect to $x$ is taken and set equal to zero from which the optimum insulation thickness $x_{o p t}$ is obtained as:

$$
x_{o p t}=293.94\left(\frac{D D C_{f} P W F \lambda}{H_{u} C_{I} \eta_{s}}\right)^{1 / 2}-\lambda R_{w t}(13)
$$

\subsection{Environmental Analysis}

The general chemical formula for the combustion of fuels is as follows:

$$
\mathrm{C}_{k} \mathrm{H}_{l} \mathrm{O}_{m} \mathrm{~S}_{p} \mathrm{~N}_{r}+\alpha A\left(\mathrm{O}_{2}+3.76 \mathrm{~N}_{2}\right) \rightarrow k \mathrm{CO}_{2}+\frac{l}{2} \mathrm{H}_{2} \mathrm{O}+\mathrm{pSO}_{2}+(\alpha-1) \mathrm{AO}_{2}+\mathrm{BN}_{2}(14)
$$
oxygen:

$A$ and $B$ can be calculated from the equilibrium formula of

$$
\begin{aligned}
& A=k+\frac{l}{4}+p-\frac{m}{2} \\
& B=3.76 \alpha\left(k+\frac{l}{4}+p-\frac{m}{2}\right)+\frac{r}{2}
\end{aligned}
$$

By ignoring $\mathrm{CO}$ and $\mathrm{NO}_{\mathrm{x}}$ emissions, the emission amounts obtained by burning one (1) $\mathrm{kg}$ of fuel can be calculated using the equation (14) as follows:

$$
\begin{aligned}
& M_{\mathrm{CO}_{2}}=\frac{k \mathrm{kO}_{2}}{M} \equiv \mathrm{kgCO}_{2} / \mathrm{kgfuel} \\
& M_{\mathrm{SO}_{2}}=\frac{p S O_{2}}{M} \equiv \mathrm{kgCO}_{2} / \mathrm{kgfuel}
\end{aligned}
$$

The total amount of $\mathrm{CO}_{2}$ and $\mathrm{SO}_{2}$ emissions can be calculated by placing the total amount of fuel $\left(m_{y A}\right)$ within the scope of $D D$ on the right side of the above equation:

$$
\begin{aligned}
& M_{\mathrm{CO}_{2}}=\frac{44 k}{M} m_{f A} \\
& M_{\mathrm{CO}_{2}}=\frac{3801600 D D k}{M \eta_{s} H_{u}}\left(\frac{\lambda}{\lambda R_{w t}+x}\right) \mathrm{kg} / \text { year } \\
& M_{\mathrm{SO}_{2}}=\frac{32 p}{M} m_{f A} \\
& M_{\mathrm{SO}_{2}}=\frac{2764800 D D p}{M \eta_{s} H_{u}}\left(\frac{\lambda}{\lambda R_{w t}+x}\right) \mathrm{kg} / \text { year }
\end{aligned}
$$

The molar weight of fuel, $M$ can be calculated as follows:

$M=12 k+l+16 m+32 p+14 r \quad \mathrm{~kg} / \mathrm{kmol}$ 


\section{FINDINGS AND DISCUSSIONS}

In this study, optimum insulation thickness and emission per unit area were calculated for building exterior walls in 81 provinces, in Turkey. Table 3-4 presents the calculation results for the insulation materials XPS and EPS. It can be seen in Table 3 that, when using the insulation material XPS, optimum insulation thickness for coal and natural gas varies between 0.031 and $0.105 \mathrm{~m}$, and 0.024 and $0.089 \mathrm{~m}$, respectively. The fuel consumed varies between 2.808 and $6.893 \mathrm{~kg} / \mathrm{m}^{2}$ per year for coal and between 1.637 and $4.020 \mathrm{~kg} / \mathrm{m}^{2}$ per year for natural gas. The $\mathrm{CO}_{2}$ emission of coal varies between 7.618 and $18.702 \mathrm{~kg} / \mathrm{m}^{2}$ per year, while $\mathrm{SO}_{2}$ emission varies between 0.007 and $0.018 \mathrm{~kg} / \mathrm{m}^{2}$ per year. The $\mathrm{CO}_{2}$ emission of natural gas varies between 4.335 and $10.642 \mathrm{~kg} / \mathrm{m}^{2}$ per year. As presented in Table 4, when using the insulation material EPS, optimum insulation thickness for coal and natural gas varies between 0.047 and $0.149 \mathrm{~m}$ and 0.038 and $0.127 \mathrm{~m}$, respectively. The amount of fuel consumed varies between 2.277 and $5.554 \mathrm{~kg} / \mathrm{m}^{2}$ per year for coal and between 1.330 and $3.239 \mathrm{~kg} / \mathrm{m}^{2}$ per year for natural gas. The $\mathrm{CO}_{2}$ emission of coal varies between 6.178 and $15.068 \mathrm{~kg} / \mathrm{m}^{2}$ per year and $\mathrm{SO}_{2}$ emission varies between 0.006 and $0.014 \mathrm{~kg} / \mathrm{m}^{2}$ per year. The $\mathrm{CO}_{2}$ emission of natural gas varies between 3.522 and $8.575 \mathrm{~kg} / \mathrm{m}^{2}$ per year. Whencoal is used; in the coldest city, $\mathrm{CO}_{2}$ and $\mathrm{SO}_{2}$ emissions were reduced by $75 \%$ when XPS were used for building insulation and by $80 \%$ when EPS was used. In the hottest city, $\mathrm{CO}_{2}$ and $\mathrm{SO}_{2}$ emissions were reduced by 50\% when XPS were used for building insulation and by $60 \%$ when EPS was used. When naturalgas is used; in the coldest city, $\mathrm{CO}_{2}$ emission was reduced by $40 \%$ when XPS was used for building insulation and by 45\% when EPS was used. In the hottest city, $\mathrm{CO}_{2}$ emission was reduced by $25 \%$ when XPS was used for building insulation and by $30 \%$ when EPS was used.

Table 3. Calculation results for 81 provinces of Turkey in case of use XPS insulation material

\begin{tabular}{|c|c|c|c|c|c|c|c|}
\hline & \multicolumn{4}{|c|}{ Coal } & \multicolumn{3}{|c|}{ Naturalgas } \\
\hline & $\mathrm{x}_{\mathrm{opt}}(\mathrm{m})$ & $\begin{array}{c}m_{f A} \\
\left(\mathrm{~kg} / \mathrm{m}^{2} \text {-year }\right)\end{array}$ & $\begin{array}{c}\mathrm{M}_{\mathrm{CO} 2} \\
\left(\mathrm{~kg} / \mathrm{m}^{2}-\text { year }\right)\end{array}$ & $\begin{array}{c}\mathrm{M}_{\mathrm{SO2}} \\
\left(\mathrm{kg} / \mathrm{m}^{2} \text {-year }\right)\end{array}$ & $x_{o p t}(m)$ & $\begin{array}{c}\mathrm{m}_{\mathrm{fA}} \\
\left(\mathrm{kg} / \mathrm{m}^{2} \text {-year }\right)\end{array}$ & $\begin{array}{c}\mathrm{M}_{\mathrm{CO} 2} \\
\left(\mathrm{~kg} / \mathrm{m}^{2}-\text { year }\right)\end{array}$ \\
\hline Adana & 0.031 & 2.841 & 7.708 & 0.007 & 0.024 & 1.658 & 4.389 \\
\hline Adıyaman & 0.052 & 3.956 & 10.735 & 0.010 & 0.042 & 2.308 & 6.111 \\
\hline Afyon & 0.073 & 5.111 & 13.866 & 0.013 & 0.061 & 2.982 & 7.895 \\
\hline Ağrı & 0.096 & 6.396 & 17.354 & 0.017 & 0.081 & 3.733 & 9.882 \\
\hline Amasya & 0.062 & 4.520 & 12.264 & 0.012 & 0.051 & 2.639 & 6.986 \\
\hline Ankara & 0.070 & 5.475 & 14.856 & 0.014 & 0.059 & 2.902 & 7.684 \\
\hline Antalya & 0.037 & 3.163 & 8.581 & 0.008 & 0.030 & 1.846 & 4.888 \\
\hline Artvin & 0.066 & 4.739 & 12.858 & 0.012 & 0.055 & 2.766 & 7.323 \\
\hline Aydın & 0.040 & 3.351 & 9.093 & 0.009 & 0.033 & 1.956 & 5.178 \\
\hline Balıkesir & 0.056 & 4.204 & 11.407 & 0.011 & 0.046 & 2.456 & 6.501 \\
\hline Bilecik & 0.065 & 4.705 & 12.765 & 0.012 & 0.054 & 2.748 & 7.274 \\
\hline Bingöl & 0.073 & 5.124 & 13.902 & 0.013 & 0.061 & 2.990 & 7.917 \\
\hline Bitlis & 0.080 & 5.535 & 15.018 & 0.014 & 0.067 & 3.228 & 8.546 \\
\hline Bolu & 0.073 & 5.105 & 13.850 & 0.013 & 0.061 & 2.980 & 7.889 \\
\hline Burdur & 0.065 & 4.662 & 12.650 & 0.012 & 0.054 & 2.720 & 7.202 \\
\hline Bursa & 0.056 & 4.212 & 11.428 & 0.011 & 0.046 & 2.460 & 6.512 \\
\hline Çanakkale & 0.054 & 4.065 & 11.029 & 0.010 & 0.044 & 2.372 & 6.279 \\
\hline Çankırı & 0.073 & 5.143 & 13.955 & 0.013 & 0.061 & 3.003 & 7.950 \\
\hline Çorum & 0.075 & 5.227 & 14.181 & 0.014 & 0.063 & 3.053 & 8.083 \\
\hline Denizli & 0.050 & 3.877 & 10.518 & 0.010 & 0.041 & 2.263 & 5.991 \\
\hline Diyarbakır & 0.061 & 4.451 & 12.077 & 0.012 & 0.050 & 2.595 & 6.871 \\
\hline Edirne & 0.062 & 4.532 & 12.298 & 0.012 & 0.052 & 2.644 & 7.001 \\
\hline Elazığ & 0.070 & 4.954 & 13.440 & 0.013 & 0.058 & 2.891 & 7.654 \\
\hline Ercincan & 0.076 & 5.309 & 14.404 & 0.014 & 0.064 & 3.095 & 8.195 \\
\hline Erzurum & 0.102 & 6.678 & 18.119 & 0.018 & 0.086 & 3.898 & 10.319 \\
\hline Eskişehir & 0.076 & 5.307 & 14.398 & 0.014 & 0.064 & 3.099 & 8.204 \\
\hline Gaziantep & 0.058 & 4.312 & 11.700 & 0.011 & 0.048 & 2.514 & 6.655 \\
\hline Giresun & 0.053 & 4.037 & 10.954 & 0.010 & 0.044 & 2.356 & 6.237 \\
\hline Gümüşhane & 0.079 & 5.466 & 14.830 & 0.014 & 0.066 & 3.189 & 8.442 \\
\hline
\end{tabular}


Aslan, A.,

Engineering Sciences, 2021, 16(2):64-77.

\begin{tabular}{|c|c|c|c|c|c|c|c|}
\hline Hakkari & 0.083 & 5.661 & 15.360 & 0.015 & 0.070 & 3.304 & 8.748 \\
\hline Hatay & 0.038 & 3.214 & 8.722 & 0.008 & 0.030 & 1.876 & 4.966 \\
\hline Isparta & 0.069 & 4.911 & 13.325 & 0.013 & 0.057 & 2.866 & 7.588 \\
\hline İçel & 0.031 & 2.808 & 7.618 & 0.007 & 0.024 & 1.637 & 4.335 \\
\hline İstanbul & 0.055 & 4.150 & 11.261 & 0.011 & 0.045 & 2.422 & 6.411 \\
\hline İzmir & 0.038 & 3.215 & 8.723 & 0.008 & 0.030 & 1.876 & 4.698 \\
\hline Kars & 0.101 & 6.640 & 18.016 & 0.017 & 0.085 & 3.875 & 10.259 \\
\hline Kastamonu & 0.077 & 5.361 & 14.546 & 0.014 & 0.065 & 3.129 & 8.285 \\
\hline Kayseri & 0.077 & 5.363 & 14.551 & 0.014 & 0.065 & 3.138 & 8.307 \\
\hline Kırklareli & 0.063 & 4.585 & 12.439 & 0.012 & 0.052 & 2.674 & 7.080 \\
\hline Kırşehir & 0.073 & 5.142 & 13.951 & 0.013 & 0.061 & 2.999 & 7.970 \\
\hline Kocaeli & 0.054 & 4.061 & 11.019 & 0.010 & 0.044 & 2.373 & 6.282 \\
\hline Konya & 0.073 & 5.118 & 13.886 & 0.013 & 0.061 & 2.988 & 7.971 \\
\hline Kütahya & 0.074 & 5.161 & 14.003 & 0.013 & 0.061 & 3.012 & 7.975 \\
\hline Malatya & 0.067 & 4.769 & 12.938 & 0.012 & 0.055 & 2.784 & 7.370 \\
\hline Manisa & 0.048 & 3.765 & 10.215 & 0.010 & 0.039 & 2.197 & 5.817 \\
\hline K.Maraş & 0.051 & 3.908 & 10.603 & 0.010 & 0.042 & 2.280 & 6.038 \\
\hline Mardin & 0.058 & 4.307 & 11.686 & 0.011 & 0.048 & 2.511 & 6.648 \\
\hline Muğla & 0.055 & 4.171 & 11.316 & 0.011 & 0.046 & 2.431 & 6.435 \\
\hline Muş & 0.084 & 5.741 & 15.577 & 0.015 & 0.071 & 3.348 & 8.865 \\
\hline Nevşehir & 0.076 & 5.292 & 14.360 & 0.014 & 0.064 & 3.088 & 8.176 \\
\hline Niğde & 0.073 & 5.140 & 13.946 & 0.013 & 0.061 & 2.998 & 7.938 \\
\hline Ordu & 0.054 & 4.084 & 11.082 & 0.011 & 0.044 & 2.382 & 6.306 \\
\hline Rize & 0.054 & 4.104 & 11.136 & 0.011 & 0.045 & 2.392 & 6.333 \\
\hline Sakarya & 0.055 & 4.117 & 11.171 & 0.011 & 0.045 & 2.402 & 6.359 \\
\hline Samsun & 0.054 & 4.107 & 11.143 & 0.011 & 0.045 & 2.396 & 6.344 \\
\hline Siirt & 0.057 & 4.257 & 11.550 & 0.011 & 0.047 & 2.482 & 6.572 \\
\hline Sinop & 0.055 & 4.166 & 11.303 & 0.011 & 0.046 & 2.431 & 6.435 \\
\hline Sivas & 0.083 & 5.640 & 15.304 & 0.015 & 0.069 & 3.294 & 8.721 \\
\hline Tekirdağ & 0.059 & 4.334 & 11.760 & 0.011 & 0.048 & 2.528 & 6.692 \\
\hline Tokat & 0.065 & 4.708 & 12.773 & 0.012 & 0.054 & 2.747 & 7.272 \\
\hline Trabzon & 0.052 & 3.994 & 10.837 & 0.010 & 0.043 & 2.330 & 6.169 \\
\hline Tunceli & 0.071 & 5.008 & 13.588 & 0.013 & 0.059 & 2.922 & 7.736 \\
\hline Şanlıurfa & 0.047 & 3.727 & 10.112 & 0.010 & 0.039 & 2.174 & 5.756 \\
\hline Uşak & 0.066 & 4.722 & 12.811 & 0.012 & 0.055 & 2.756 & 7.297 \\
\hline $\operatorname{Van}$ & 0.083 & 5.671 & 15.387 & 0.015 & 0.070 & 3.306 & 8.752 \\
\hline Yozgat & 0.082 & 5.626 & 15.265 & 0.015 & 0.069 & 3.280 & 8.684 \\
\hline Zonguldak & 0.058 & 4.319 & 11.720 & 0.011 & 0.048 & 2.520 & 6.672 \\
\hline Aksaray & 0.069 & 4.925 & 13.362 & 0.013 & 0.058 & 2.876 & 7.614 \\
\hline Bayburt & 0.093 & 6.190 & 16.796 & 0.016 & 0.074 & 3.612 & 9.562 \\
\hline Karaman & 0.071 & 4.992 & 13.544 & 0.013 & 0.059 & 2.913 & 7.711 \\
\hline Kırıkkale & 0.069 & 4.909 & 13.320 & 0.013 & 0.058 & 2.865 & 7.584 \\
\hline Batman & 0.054 & 4.106 & 11.140 & 0.011 & 0.045 & 2.396 & 6.343 \\
\hline Şırnak & 0.049 & 4.367 & 11.849 & 0.011 & 0.049 & 2.548 & 6.717 \\
\hline Bartın & 0.062 & 4.534 & 12.303 & 0.012 & 0.052 & 2.647 & 7.007 \\
\hline Ardahan & 0.105 & 6.893 & 18.702 & 0.018 & 0.089 & 4.020 & 10.642 \\
\hline Iğdır & 0.072 & 5.055 & 13.715 & 0.013 & 0.060 & 2.948 & 7.806 \\
\hline Yalova & 0.055 & 4.129 & 11.202 & 0.011 & 0.045 & 2.407 & 6.374 \\
\hline Karabük & 0.061 & 4.452 & 12.079 & 0.012 & 0.050 & 2.623 & 6.944 \\
\hline Kilis & 0.049 & 3.792 & 10.290 & 0.010 & 0.040 & 2.215 & 5.864 \\
\hline Osmaniye & 0.038 & 3.192 & 8.660 & 0.008 & 0.030 & 1.866 & 4.939 \\
\hline Düzce & 0.059 & 4.371 & 11.861 & 0.011 & 0.049 & 2.550 & 6.752 \\
\hline
\end{tabular}

Table 4. Calculation results for 81 provinces of Turkey in case of use EPS insulation material

\begin{tabular}{|l|l|c|c|c|c|c|c|}
\hline \multirow{2}{*}{} & \multicolumn{3}{|c|}{$\begin{array}{c}\text { Coal } \\
\text { Naturalgas }\end{array}$} \\
\cline { 2 - 8 } & $x_{\text {opt }}(\mathrm{m})$ & $\begin{array}{c}\mathrm{m}_{\mathrm{fA}} \\
\left(\mathrm{kg} / \mathrm{m}^{2}-\text { year }\right)\end{array}$ & $\begin{array}{c}\mathrm{M}_{\mathrm{CO} 2} \\
\left(\mathrm{~kg} / \mathrm{m}^{2}-\mathrm{year}\right)\end{array}$ & $\begin{array}{c}\mathrm{M}_{\mathrm{SO2}} \\
\left(\mathrm{kg} / \mathrm{m}^{2}-\mathrm{year}\right)\end{array}$ & $\mathrm{x}_{\text {opt }}(\mathrm{m})$ & $\begin{array}{c}\mathrm{m}_{\mathrm{fA}} \\
\left(\mathrm{kg} / \mathrm{m}^{2}-\mathrm{year}\right)\end{array}$ & $\begin{array}{c}\mathrm{M}_{\mathrm{CO} 2} \\
\left(\mathrm{~kg} / \mathrm{m}^{2}-\mathrm{year}\right)\end{array}$ \\
\hline Adana & 0.048 & 2.302 & 6.247 & 0.006 & 0.039 & 1.342 & 3.554 \\
\hline Adıyaman & 0.076 & 3.174 & 8.612 & 0.008 & 0.063 & 1.853 & 4.906 \\
\hline Afyon & 0.105 & 4.101 & 11.128 & 0.011 & 0.089 & 2.392 & 6.332 \\
\hline Ağrı & 0.137 & 5.129 & 13.916 & 0.013 & 0.116 & 2.993 & 7.924 \\
\hline Amasya & 0.090 & 3.624 & 9.833 & 0.009 & 0.076 & 2.116 & 5.603 \\
\hline Ankara & 0.102 & 3.988 & 10.822 & 0.010 & 0.085 & 2.327 & 6.161 \\
\hline Antalya & 0.056 & 2.561 & 6.950 & 0.006 & 0.046 & 1.493 & 3.952 \\
\hline Artvin & 0.096 & 3.802 & 10.317 & 0.010 & 0.080 & 2.235 & 5.917 \\
\hline Aydın & 0.062 & 2.704 & 7.338 & 0.007 & 0.050 & 1.597 & 4.181 \\
\hline Balıkesir & 0.082 & 3.372 & 9.150 & 0.009 & 0.069 & 1.969 & 5.213 \\
\hline Bilecik & 0.095 & 3.774 & 10.239 & 0.010 & 0.080 & 2.202 & 5.830 \\
\hline
\end{tabular}


Aslan, A.,

Engineering Sciences, 2021, 16(2):64-77.

\begin{tabular}{|c|c|c|c|c|c|c|c|}
\hline Bingöl & 0.105 & 4.129 & 11.202 & 0.011 & 0.089 & 2.400 & 6.355 \\
\hline Bitlis & 0.115 & 4.437 & 12.038 & 0.011 & 0.098 & 2.591 & 6.859 \\
\hline Bolu & 0.105 & 4.094 & 11.109 & 0.011 & 0.088 & 2.390 & 6.328 \\
\hline Burdur & 0.094 & 3.737 & 10.140 & 0.010 & 0.079 & 2.181 & 5.773 \\
\hline Bursa & 0.082 & 3.409 & 9.250 & 0.009 & 0.069 & 1.980 & 5.241 \\
\hline Çanakkale & 0.079 & 3.271 & 8.874 & 0.008 & 0.066 & 1.903 & 5.038 \\
\hline Çankırı & 0.106 & 4.134 & 11.217 & 0.011 & 0.089 & 2.422 & 6.413 \\
\hline Çorum & 0.108 & 4.204 & 11.407 & 0.011 & 0.091 & 2.458 & 6.507 \\
\hline Denizli & 0.074 & 3.129 & 8.490 & 0.008 & 0.062 & 1.817 & 4.810 \\
\hline Diyarbakır & 0.088 & 3.570 & 9.688 & 0.009 & 0.074 & 2.083 & 5.515 \\
\hline Edirne & 0.091 & 3.635 & 9.863 & 0.009 & 0.076 & 2.130 & 5.639 \\
\hline Elazığ & 0.101 & 3.985 & 10.812 & 0.010 & 0.085 & 2.327 & 6.162 \\
\hline Ercincan & 0.110 & 4.265 & 11.573 & 0.011 & 0.093 & 2.488 & 6.586 \\
\hline Erzurum & 0.144 & 5.375 & 14.585 & 0.014 & 0.123 & 3.127 & 8.279 \\
\hline Eskişehir & 0.110 & 4.268 & 11.580 & 0.011 & 0.093 & 2.489 & 6.590 \\
\hline Gaziantep & 0.085 & 3.467 & 9.408 & 0.009 & 0.071 & 2.016 & 5.338 \\
\hline Giresun & 0.078 & 3.259 & 8.842 & 0.008 & 0.065 & 1.903 & 5.039 \\
\hline Gümüşhane & 0.114 & 4.394 & 11.922 & 0.011 & 0.096 & 2.573 & 6.813 \\
\hline Hakkari & 0.119 & 4.548 & 12.340 & 0.012 & 0.100 & 2.671 & 7.071 \\
\hline Hatay & 0.058 & 2.581 & 7.003 & 0.006 & 0.047 & 1.520 & 4.024 \\
\hline Isparta & 0.100 & 3.948 & 10.711 & 0.010 & 0.084 & 2.298 & 6.084 \\
\hline İçel & 0.047 & 2.277 & 6.178 & 0.006 & 0.038 & 1.330 & 3.522 \\
\hline İstanbul & 0.081 & 3.331 & 9.037 & 0.000 & 0.068 & 1.944 & 5.147 \\
\hline İzmir & 0.058 & 2.578 & 6.996 & 0.006 & 0.047 & 1.519 & 4.021 \\
\hline Kars & 0.143 & 5.346 & 14.506 & 0.014 & 0.122 & 3.113 & 8.242 \\
\hline Kastamonu & 0.111 & 4.323 & 11.730 & 0.011 & 0.094 & 2.510 & 6.646 \\
\hline Kayseri & 0.111 & 4.325 & 11.734 & 0.011 & 0.094 & 2.511 & 6.648 \\
\hline Kırklareli & 0.092 & 3.684 & 9.997 & 0.009 & 0.077 & 2.156 & 5.707 \\
\hline Kırşehir & 0.106 & 4.124 & 11.189 & 0.011 & 0.089 & 2.416 & 6.397 \\
\hline Kocaeli & 0.079 & 3.265 & 8.859 & 0.008 & 0.066 & 1.904 & 5.041 \\
\hline Konya & 0.105 & 4.126 & 11.194 & 0.011 & 0.089 & 2.399 & 6.350 \\
\hline Kütahya & 0.106 & 4.157 & 11.279 & 0.011 & 0.090 & 2.414 & 6.391 \\
\hline Malatya & 0.096 & 3.827 & 10.383 & 0.010 & 0.081 & 2.243 & 5.937 \\
\hline Manisa & 0.071 & 3.021 & 8.197 & 0.008 & 0.059 & 1.777 & 4.706 \\
\hline K.Maraş & 0.075 & 3.146 & 8.537 & 0.008 & 0.062 & 1.846 & 4.887 \\
\hline Mardin & 0.085 & 3.459 & 9.385 & 0.009 & 0.071 & 2.022 & 5.353 \\
\hline Muğla & 0.081 & 3.369 & 9.140 & 0.009 & 0.068 & 1.959 & 5.186 \\
\hline Muş & 0.121 & 4.605 & 12.493 & 0.012 & 0.102 & 2.698 & 7.144 \\
\hline Nevşehir & 0.121 & 3.920 & 10.635 & 0.010 & 0.102 & 2.297 & 6.081 \\
\hline Niğde & 0.106 & 4.122 & 11.185 & 0.011 & 0.089 & 2.415 & 6.395 \\
\hline Ordu & 0.079 & 3.298 & 8.949 & 0.008 & 0.066 & 1.923 & 5.092 \\
\hline Rize & 0.080 & 3.295 & 8.940 & 0.008 & 0.066 & 1.940 & 5.137 \\
\hline Sakarya & 0.080 & 3.318 & 9.004 & 0.008 & 0.067 & 1.932 & 5.116 \\
\hline Samsun & 0.080 & 3.306 & 8.969 & 0.008 & 0.067 & 1.925 & 5.096 \\
\hline Siirt & 0.084 & 3.411 & 9.256 & 0.009 & 0.070 & 1.997 & 5.287 \\
\hline Sinop & 0.081 & 3.369 & 9.140 & 0.009 & 0.068 & 1.959 & 5.186 \\
\hline Sivas & 0.118 & 4.546 & 12.334 & 0.012 & 0.100 & 2.651 & 7.018 \\
\hline Tekirdağ & 0.086 & 3.475 & 9.428 & 0.009 & 0.072 & 2.028 & 5.370 \\
\hline Tokat & 0.095 & 3.788 & 10.277 & 0.010 & 0.080 & 2.207 & 5.844 \\
\hline Trabzon & 0.077 & 3.215 & 8.724 & 0.008 & 0.064 & 1.880 & 4.979 \\
\hline Tunceli & 0.102 & 4.047 & 10.979 & 0.010 & 0.086 & 2.361 & 6.250 \\
\hline Şanlıurfa & 0.070 & 3.016 & 8.183 & 0.008 & 0.058 & 1.762 & 4.665 \\
\hline Uşak & 0.095 & 3.811 & 10.341 & 0.010 & 0.080 & 2.221 & 5.881 \\
\hline Van & 0.119 & 4.556 & 12.360 & 0.012 & 0.101 & 2.654 & 7.026 \\
\hline Yozgat & 0.118 & 4.517 & 12.256 & 0.012 & 0.100 & 2.634 & 6.973 \\
\hline Zonguldak & 0.085 & 3.486 & 9.460 & 0.009 & 0.071 & 2.038 & 5.396 \\
\hline Aksaray & 0.100 & 3.976 & 10.789 & 0.010 & 0.084 & 2.325 & 6.157 \\
\hline Bayburt & 0.132 & 4.980 & 13.511 & 0.013 & 0.112 & 2.908 & 7.699 \\
\hline Karaman & 0.102 & 4.020 & 10.907 & 0.010 & 0.086 & 2.345 & 6.209 \\
\hline Kırıkkale & 0.100 & 3.951 & 10.729 & 0.010 & 0.084 & 2.311 & 6.117 \\
\hline Batman & 0.080 & 3.300 & 8.954 & 0.008 & 0.067 & 1.922 & 5.088 \\
\hline Şırnak & 0.086 & 3.531 & 9.581 & 0.009 & 0.072 & 2.061 & 5.458 \\
\hline Bartin & 0.091 & 3.638 & 9.872 & 0.009 & 0.076 & 2.132 & 5.644 \\
\hline Ardahan & 0.149 & 5.554 & 15.068 & 0.014 & 0.127 & 3.239 & 8.575 \\
\hline Iğdır & 0.104 & 4.053 & 10.996 & 0.010 & 0.087 & 2.380 & 6.302 \\
\hline Yalova & 0.080 & 3.336 & 9.053 & 0.009 & 0.067 & 1.943 & 5.144 \\
\hline Karabük & 0.088 & 3.601 & 9.772 & 0.009 & 0.074 & 2.097 & 5.551 \\
\hline Kilis & 0.072 & 3.052 & 8.281 & 0.008 & 0.060 & 1.777 & 4.706 \\
\hline Osmaniye & 0.575 & 2.560 & 6.946 & 0.006 & 0.047 & 1.498 & 3.967 \\
\hline Düzce & 0.086 & 3.531 & 9.581 & 0.009 & 0.072 & 2.061 & 5.458 \\
\hline
\end{tabular}


However, one province from each of five climate zones in Turkey, including Antalya, Istanbul, Ankara Kayseri and Erzurum, were selected and compared for heating and cooling seasons. Fuel and electricity costs were determined, and optimum insulation thickness, net energy conservations and annual payback periods were calculated for each province. The effect of using insulation materials on annual combustion products were examined depending on insulation thickness. Figure 1 gives the optimum points indicating minimum total cost for heating and cooling loads in Antalya, Istanbul, Ankara, Kayseri and Erzurum. As insulation thickness increases, heating load and fuel cost decrease, but insulation cost increases. However, the total cost of fuel and insulation decreases up to a point, then increases again. The point referring to the minimum total cost indicates the optimum insulation thickness.
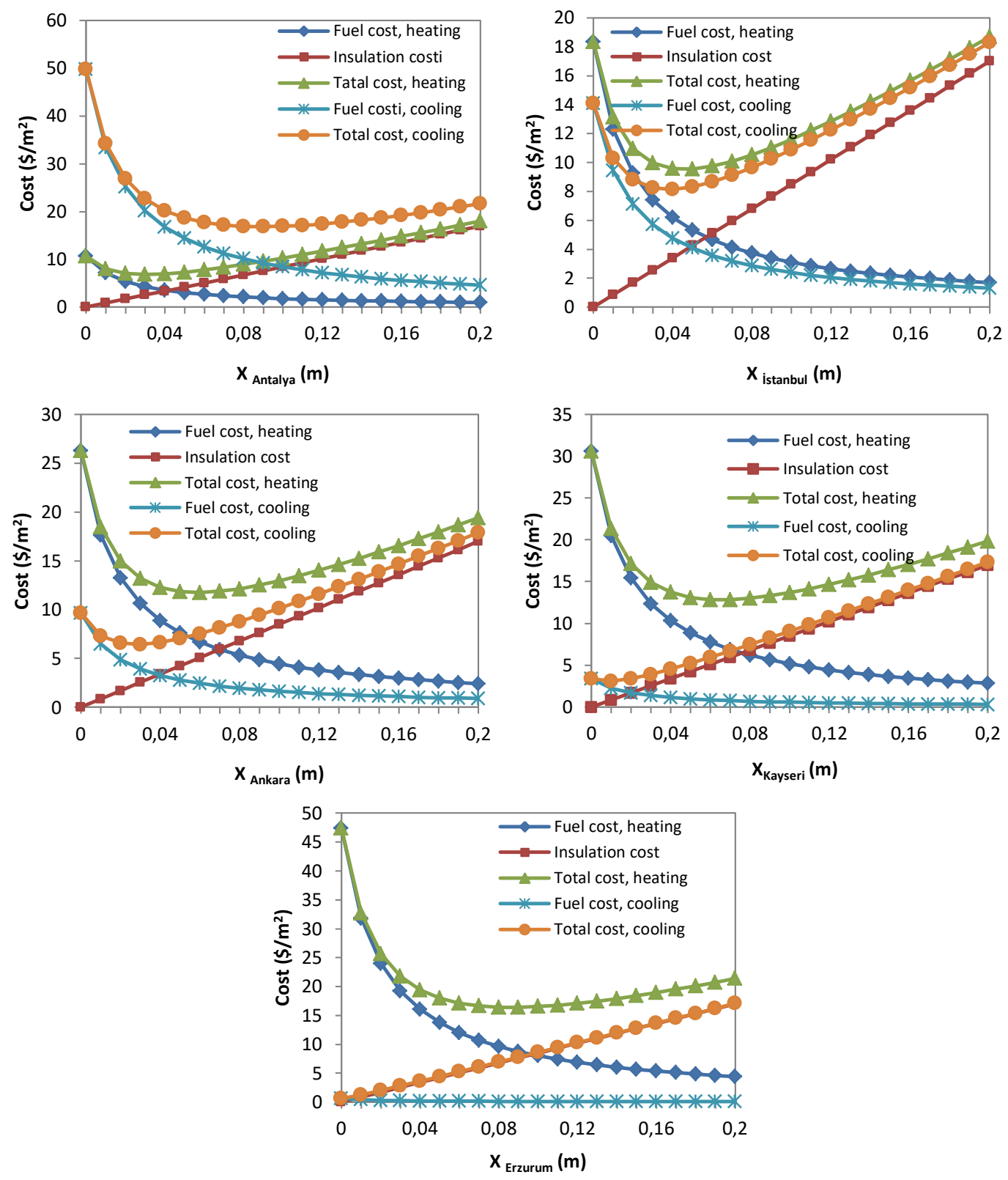

Figure 1. Optimum insulation thickness in heating and cooling 
Figure 2 shows the effect of annual energy conservations on insulation thickness for different energy sources (natural gas and coal) in cause of use of XPS and EPS insulation materials in heating. A higher energy conservation was obtained when coal was used as fuel type. The highest energy conservation was achieved in Erzurum, which is the coldest province in all cases, while the lowest energy conservations was achieved in Antalya, the hottest province.
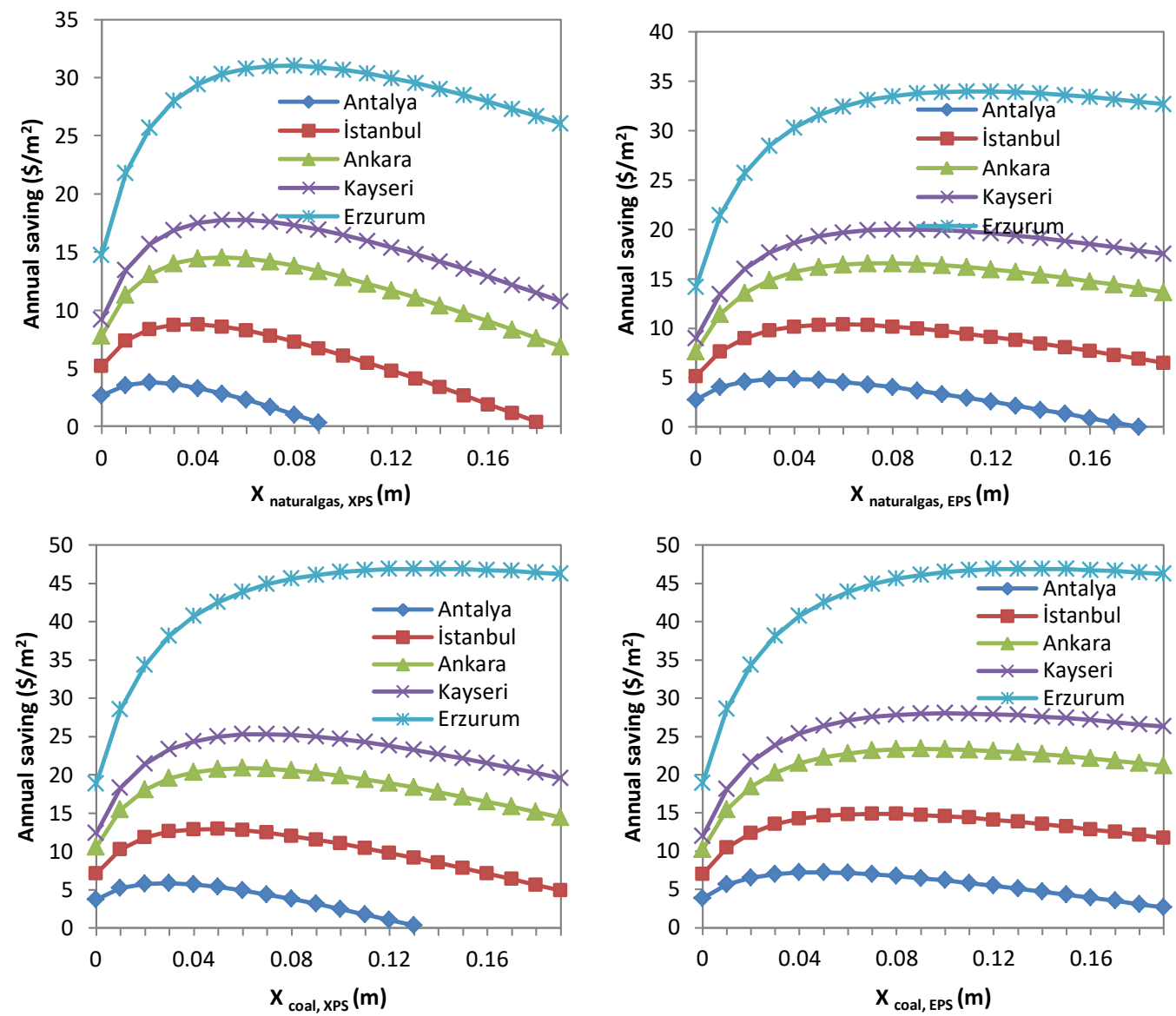

Figure 2. The effect of annual savings on insulation thickness

Table 5 presents the values recorded at the optimum point for each province, and gives the energy conservation calculated per unit area and the payback period values in case of application of optimum insulation thickness for different insulation materials and fuel types on external walls. The optimum insulation thickness varied according to type of fuels used in heating (natural gas, coal) and cooling (electricity) and insulation materials (XPS and EPS). As expected, the maximum energy conservation and the shortest payback period were achieved in the same case. The shortest payback periods for heating and cooling were obtained in Erzurum and Antalya, respectively. A higher energy conservation was achieved when EPS and coal were used in Erzurum and when EPS and coal were used in Antalya. By using XPS insulation material for climatic conditions in Antalya, the optimum insulation thicknesses were obtained as $0.030 \mathrm{~m}$ for heating with natural gas and $0.051 \mathrm{~m}$ for cooling with electricity. These values were found as 0.045 and 0.018 for Istanbul, 0.059 and 0.011 for Ankara, 0.065 and $<0$ for Kayseri and 0.086 and $<0$ for Erzurum. In the case of using EPS insulation material, higher values were obtained in all provinces. 
Table 5. Optimum values, savings and payback period in heating and cooling

\begin{tabular}{|c|c|c|c|c|c|c|c|c|}
\hline \multirow[b]{2}{*}{ 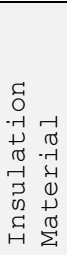 } & \multicolumn{4}{|c|}{ Heating } & \multicolumn{4}{|c|}{ Cooling } \\
\hline & Fuel & $\mathrm{x}_{\mathrm{opt}}(\mathrm{m})$ & $\begin{array}{c}\text { Energy } \\
\text { saving } \\
\left(\$ / \mathrm{m}^{2}\right)\end{array}$ & $\begin{array}{c}\text { Payback } \\
\text { period } \\
\text { (years) }\end{array}$ & Fuel & $\mathrm{x}_{\mathrm{opt}}(\mathrm{m})$ & $\begin{array}{c}\text { Energy } \\
\text { Saving } \\
\left(\$ / \mathrm{m}^{2}\right)\end{array}$ & $\begin{array}{c}\text { Payback } \\
\text { Period } \\
\text { (years) }\end{array}$ \\
\hline \multicolumn{9}{|c|}{ Antalya } \\
\hline \multirow{2}{*}{ XPS } & NaturalGas & 0.030 & 3.78 & 2.80 & \multirow{2}{*}{ Electricity } & \multirow{2}{*}{0.051} & \multirow{2}{*}{11.19} & \multirow{2}{*}{1.94} \\
\hline & Coal & 0.037 & 5.89 & 2.37 & & & & \\
\hline \multirow{2}{*}{ EPS } & NaturalGas & 0.046 & 4.87 & 2.18 & \multirow{2}{*}{ Electricity } & \multirow[b]{2}{*}{0.076} & \multirow[b]{2}{*}{13.00} & \multirow[b]{2}{*}{1.67} \\
\hline & Coal & 0.056 & 7.23 & 1.93 & & & & \\
\hline \multicolumn{9}{|c|}{ İstanbul } \\
\hline \multirow{2}{*}{ XPS } & NaturalGas & 0.045 & 8.79 & 2.08 & \multirow{2}{*}{ Electricity } & \multirow{2}{*}{0.018} & \multirow{2}{*}{1.35} & \multirow{2}{*}{4.53} \\
\hline & Coal & 0.055 & 12.94 & 1.86 & & & & \\
\hline \multirow{2}{*}{ EPS } & NaturalGas & 0.068 & 10.40 & 1.76 & \multirow{2}{*}{ Electricity } & \multirow{2}{*}{0.030} & \multirow{2}{*}{2.02} & \multirow{2}{*}{3.03} \\
\hline & Coal & 0.081 & 14.89 & 1.62 & & & & \\
\hline \multicolumn{9}{|c|}{ Ankara } \\
\hline \multirow{2}{*}{ XPS } & NaturalGas & 0.059 & 14.54 & 1.80 & \multirow{2}{*}{ Electricity } & \multirow{2}{*}{0.011} & \multirow{2}{*}{0.54} & \multirow{2}{*}{7.75} \\
\hline & Coal & 0.070 & 20.90 & 1.65 & & & & \\
\hline$F P C_{1}$ & NaturalGas & 0.085 & 16.59 & 1.58 & & & & \\
\hline EPS & Coal & 0.102 & 23.35 & 1.48 & Elect & 0.021 & 0.99 & 4.23 \\
\hline & & & & Kayse & & & & \\
\hline $\mathrm{YPC}$ & NaturalGas & 0.065 & 17.76 & 1.72 & Eloctrigitu & $<0$ & 0.01 & $E_{-}$ \\
\hline XPS & Coal & 0.077 & 25.33 & 1.59 & Electricity & $<0$ & 0.01 & - \\
\hline FPS & NaturalGas & 0.094 & 20.03 & 1.52 & Floctrigity & $0 \cap \cap 03$ & $0=0$ & - \\
\hline EPS & Coal & 0.111 & 28.02 & 1.43 & Electricity & 0.003 & 0.02 & - \\
\hline & & & & Erzur & & & & \\
\hline XPS & NaturalGas & 0.086 & 31.04 & 1.52 & & $<0$ & $-{ }^{-}$ & - \\
\hline XPS & Coal & 0.102 & 43.43 & 1.43 & Electricity & $<0$ & - & - \\
\hline FPS & NaturalGas & 0.123 & 34.01 & 1.39 & igito & & - & - \\
\hline EPS & Coal & 0.144 & 46.93 & 1.33 & Electricity & $<0$ & - & - \\
\hline
\end{tabular}

Figure 3 shows the changes of annual fuel consumption and $\mathrm{CO}_{2}$ and $\mathrm{SO}_{2}$ emissions in heating with coal according to insulation thickness. As insulation thickness increased, annual fuel consumption and greenhouse gas emissions decreased. Although this decrease varied slightly according to the type of insulation material, it has become horizontal after a point. The highest and lowest values were obtained in Erzurum and Antalya, respectively. 

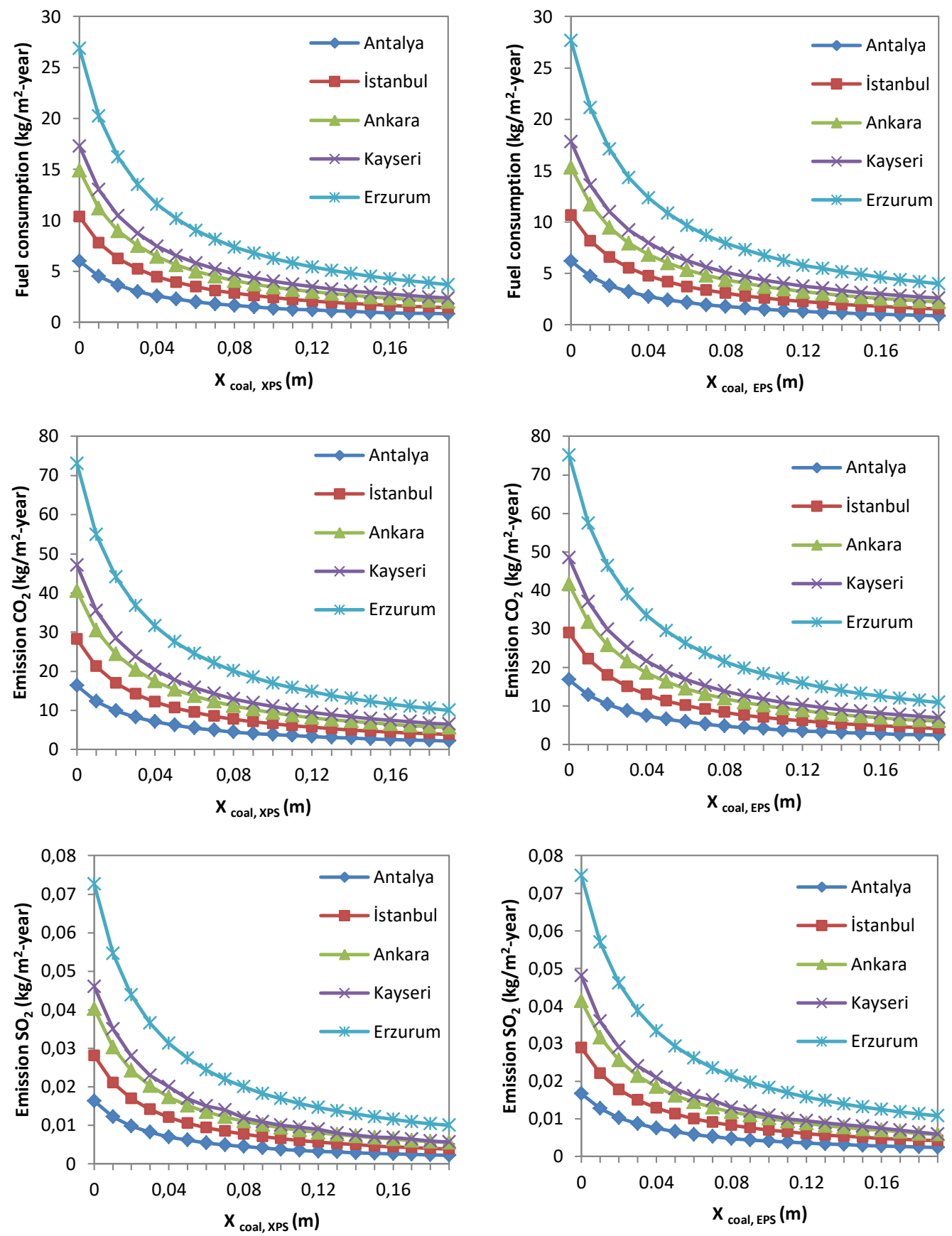

Figure 3.Fuel consumption and emissions

\section{CONCLUSION}

The application of optimum insulation thickness in buildings is essential for reducing fuel consumption and emissions. In this study, the optimum insulation thickness and emission values per unit area were calculated for building exterior walls located in 81 provinces. As the outdoor temperature decreases, the impact of insulation thickness on energy saving and emission becomes more apparent. It was observed that a maximum 75\%-80\% reduction of emission can be achieved depending on the insulation thickness.

However, one province from five different climatic zones in Turkey (Antalya, Istanbul, Ankara, Kayseri, Erzurum) were compared and the following results were obtained. The optimum insulation 
thicknesses were compared in all zones, and the optimum insulation thickness for cooling was higher in Antalya, the hottest province, while the optimum insulation thickness for heating was higher in other regions. The value of optimum insulation thickness for cooling was negative in Erzurum. Approximately 7 times higher energy conservation was achieved in Erzurum, the coldest province, compared to Antalya, the hottest province. In addition, the optimum insulation thickness and energy conservation in all provinces were higher for coal than natural gas. Since XPS has lower thermal conductivity, the optimum insulation thicknesses obtained in all provinces were lower for XPS than EPS. In conclusion, a significant energy conservation can be achieved through optimum insulation thickness of external walls in buildings. Due to increased energy demand and insufficiency of existing resources to meet it, it is extremely important and indispensable for Turkey to prevent energy wastes by using natural resources more efficiently and to prevent environmental pollution by reducing energy costs and fuel consumption, thus to create a positive effect against global warming.

\section{CONFLICT OF INTEREST}

The author declared no conflict of interest.

\section{FINANCIAL DISCLOSURE}

The author declare that this study has received no financial support.

\section{DECLARATION OF ETHICAL STANDARDS}

The author of this article declare that the materials and methods used in this study do not require ethical committee permission and/or legal-special permission.

\section{REFERENCES}

[1] Kurekci, N.A., (2016). Determination of optimum insulation thickness for building walls byusing heating and cooling degreeday values of all Turkey's provincial centers. Energy and Buildings, 118:197-213.

[2] Acıkkalp, E. and Yerel Kandemir, S., (2019). A method for determining optimum insulation thickness: combined economic and environmental method. Thermal Science and Engineering Progress, $11: 249-253$.

[3] Alsayed, M.F. and Tayeh, R.A., (2019). Life cycle cost analysis for determining optimal insulation thickness in palestinian buildings. Journal of Building Engineering, 22:101-112.

[4] Şencan Şahin, A., Kovacı, T., and Dikmen, E., (2020). Determination and economic analysis of the optimum insulation thickness of building walls, taking into account the annual $\mathrm{CO}_{2}$ emission. Pamukkale Univ Engineering Science Journal, In Print: PAJES-03083 DOI: 10.5505/pajes.2020.

[5] Karakaya, H., (2018). Determination of optimum insulation thickness in different walland fuel types in heating and cooling and environmental effects. Fırat Üniv. Engineering Science Journal, $30(2): 193-202$.

[6] Ozel, M., (2013). Thermal, economical and environmental analysis of Insulated building walls in a cold climate. Energy Conversion and Management, 76:674-684.

[7] Küçüktopcu, E. and Cemek, B., (2018). A study on environmental impact of insulation thickness of poultry building walls. Energy, 150:583-590. 
[8] Nyers, J., Kajtarb, L., Tomic, S., and Nyers, A., (2015). Investment-savings method for energy-economic optimization ofexternal wall thermal insulation thickness. Energy and Buildings, 86:268-274.

[9] Rakshit, D. and Muddu, D.M., (2021). Gowda, anthony james robinson, aimee byrne, optimisation of retrofit wall insulation: an ırish case study. Energy and Buildings, 235:110720.

[10] Yuan, J., Farnham, C., and Emura, K., (2017). Optimal combination of thermal resistance of insulation materials and primary fuel sources for six climate zones of japan, Energy and Buildings, 153:403-411.

[11] Xiangwei, L., Youming, Hua, G., Fazio, P., Guojie, C., and Xingguo, G., (2015). Guoda, determination of optimum insulation thickness for building walls with moisture transfer in hot summer and cold winter zone of china. Energy and Buildings, $109: 361-368$.

[12] Sabapathy, K.A. and Gedupudi, S., (2020). On the influence of concrete-straw-plaster envelope thermal mass on the cooling and heating loads for different climatic zones of India. Journal of Cleaner Production, 276:123-117.

[13] Hasan, A., (1999). Optimizing insulation thickness for buildings using life cycle cost. Applied Energy. 63(2):115-124.

[14] Kanakli, O., (2008). A Study on residential heating energy requirement and optimum insulation thickness. Renewable Energy, 33:1164-1172.

[15] Ucar, A. and Balo, F., (2010). Determination of the energy savings and the optimum insulation thickness in the four different insulated exterior walls. Renewable Energy, 35:88-94.

[16] http://www.canakkalegaz.com.tr/turkish/2019. Date of Access: 06.11 .2020 .

[17] www.dosider.org, Fuel prices, 2020. Date of Access: 06.11.2020.

[18] http://www.izocam.com.tr, Insulation Unit Prices, 2020. Date of Access: 06.11 .2020 .

[19] The Central Bank of the Republic of Turkey (TCMB), (2020), http://www.tcmb.gov.tr/. Date of Access: 06.11.2020.

[20] Turkish Statistical Institute (TUIK), (2020), http://www.tuik.gov.tr/. Date of Access: 06.11.2020. 